\title{
Dysbiosis caused by vitamin D receptor deficiency confers colonization resistance to Citrobacter rodentium through modulation of innate lymphoid cells
}

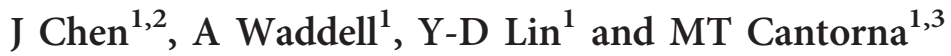

Vitamin D receptor (VDR) knockout (KO) mice had fewer Citrobacter rodentium in the feces than wild-type (WT) mice and the kinetics of clearance was faster in VDR KO than WT mice. VDR KO mice had more interleukin-22 (IL-22)-producing innate lymphoid cells (ILCs) and more antibacterial peptides than WT mice. The increased ILCs in the VDR KO mice was a cell-autonomous effect of VDR deficiency on ILC frequencies. Bone marrow (BM) transplantation from VDR KO mice into WT resulted in higher ILCs and colonization resistance of the WT mice. Disruption of the gut microbiota using antibiotics in VDR KO mice reversed colonization resistance to C. rodentium infection. Confirming the role of the microbiota in the colonization resistance of VDR KO mice, transfer of the VDR KO microbiota to WT germ-free mice resulted in colonization resistance. Once colonization resistance was overcome, VDR KO mice had increased susceptibility to C. rodentium. VDR expression is a regulator of ILC frequencies, IL-22, dysbiosis, and C. rodentium susceptibility.

\section{INTRODUCTION}

All cells of the immune system that have been analyzed express the vitamin $\mathrm{D}$ receptor (VDR) including dendritic cells, macrophages, T cells, and B cells. ${ }^{1}$ The VDR is a nuclear receptor that is part of the steroid hormone superfamily of receptors that regulate gene transcription. The active form of vitamin $\mathrm{D}$ and the high-affinity VDR ligand is 1,25-dihydroxyvitamin $\mathrm{D}_{3}\left(1,25(\mathrm{OH})_{2} \mathrm{D}_{3}\right)$., ${ }^{2}$ The effects of $1,25(\mathrm{OH})_{2} \mathrm{D}_{3}$ include direct effects on interferon- $\gamma$ and interleukin-17 (IL17) production by $T$ cells and indirect effects of $1,25(\mathrm{OH})_{2} \mathrm{D}_{3}$ on dendritic cells and macrophages that reduce $\mathrm{T}$ helper type 1 (Th1) and Th17 responses in vivo. ${ }^{4}$ The effects of vitamin D include inhibition of the generation of Th1 and Th17 responses, induction of regulatory $\mathrm{T}$ cells, and amelioration of experimental immune-mediated disease.

Because of the suppressive effects of $1,25(\mathrm{OH})_{2} \mathrm{D}_{3}$ on Th1 and Th17 responses, it seemed possible that vitamin D might compromise the anti-infectious response. Paradoxically, when therapeutic doses of $1,25(\mathrm{OH})_{2} \mathrm{D}_{3}$ (for immune-mediated disease) were tested, there was no effect of the $1,25(\mathrm{OH})_{2} \mathrm{D}_{3}$ treatment on the ability of the host to fight a Herpes simplex or Candida albicans infection. ${ }^{5}$ In addition, vitamin D supplementation of humans during tuberculosis treatment was shown to be beneficial in some studies and not to have an effect in others. ${ }^{6-9}$ In vitro and in vivo vitamin $\mathrm{D}$ induced the production of antibacterial peptides that might be beneficial in protecting the host from infection. ${ }^{10,11}$ VDR knockout (KO) mice infected with Schistosoma mansoni showed no effect of VDR expression on worm burdens, weight, and fibrosis compared with wildtype (WT) mice. ${ }^{12}$ VDR -KO mice had decreased Leishmania major parasite burdens compared with WT controls. ${ }^{13}$ VDR KO mice exhibited a delayed clearance of Listeria monocytogenes following infection but they were able to clear the infection. ${ }^{14}$ How vitamin D could inhibit Th1 and Th17 responses in one context (immune-mediated disease) but not another (infectious disease) has not been established.

Citrobacter rodentium is a Gram-negative murine pathogen that naturally colonizes and infects mice and forms attaching

\footnotetext{
${ }^{1}$ Department of Veterinary and Biomedical Science, The Pennsylvania State University, University Park, Pennsylvania, USA. ${ }^{2}$ Pathobiology Graduate Program, The Pennsylvania State University, University Park, Pennsylvania, USA and ${ }^{3}$ Center for Molecular Immunology and Infectious Disease, The Pennsylvania State University, University Park, Pennsylvania, USA. Correspondence: MT Cantorna (mxc69@psu.edu)
} 
and effacing lesions. ${ }^{15}$ In WT mice, $C$. rodentium colonizes the cecum and colon transiently and is cleared over 3 weeks. ${ }^{16}$ Th17-mediated immune responses are required for clearing the infection. ${ }^{17} \mathrm{~T}$ cell, B cell, and T and B cell (Rag) KO mice fail to clear C. rodentium infection. ${ }^{16}$ In the gut, innate lymphoid cells (ILCs) that produce IL-22 and IL-17 (ILC3) are critical for early protection against $C$. rodentium infection. ${ }^{17}$ ILC3 cells are the major source of IL-22 during the first 6 days following infection. ${ }^{18}$ The innate immune system induces the development of protective acquired immunity required for clearance of C. rodentium.

Here we determined the role of the VDR on host resistance to C. rodentium infection. Surprisingly, VDR KO mice were resistant to colonization with $C$. rodentium. The colonization resistance of the VDR KO mice was associated with increased IL-22, increased ILCs, and higher expression of RegIII $\gamma$, angiogenin-4 (Ang-4), and dysbiosis of the bacterial microbiota. Antibiotic (ABX) disruption of the gut microbiota reversed colonization resistance to $C$. rodentium in VDR KO mice. Cecal transplantation of the microbiota from VDR KO mice to WT germ-free (GF) mice conferred colonization resistance compared with recipients of WT microbiota. In addition, VDR KO bone marrow (BM) transplantation transferred colonization resistance to WT mice, associated with increased IL-22 and increased ILCs. Double VDR/Rag (D)KO mice had increased ILCs and IL-22 production in the gut. However, colonization resistance was not observed in the DKO mice. Both the DKO mice and the ABX-treated VDR-KO mice developed more severe infections, including increased mortality, than their respective controls (Rag KO and ABX WT) to $C$. rodentium infection. Our data demonstrate that increased IL-22-producing ILCs contribute to dysbiosis and C. rodentium colonization resistance of VDR KO mice. Taken together, our data suggest that VDR regulates gut microbiota, colonization, and susceptibility to C. rodentium.

\section{RESULTS}

Reduced bacterial shedding and inflammation in VDR KO mice

In WT mice, $C$. rodentium was detectable in the feces at day 1 (d1) and increased through $\mathrm{d} 7$ when it peaked and then declined until d21 (Figure 1a). At d1 after infection, VDR KO mice had 3 logs fewer bacteria in the feces than WT mice (Figure 1a). The bacterial shedding in the feces was lower in VDR KO mice compared with WT, and the VDR KO mice cleared the infection by d18 (Figure 1a). VDR KO mice were less susceptible to $C$. rodentium than WT mice (Figure 1a). VDR KO mice were resistant and WT mice were susceptible to C. rodentium infection, regardless of ancestry (breeders), sex, or housing of the mice.

The recruitment of inflammatory cells into the colonic lamina propria (LP) was measured in VDR KO and WT mice. In uninfected $(\mathrm{d} 0)$ WT and VDR KO mice, the frequencies of either inflammatory monocytes $\left(\mathrm{CD} 11 \mathrm{~b}^{+} \mathrm{Gr}-\mathrm{1}^{\text {high }} \mathrm{F} 4 / 80^{+}\right.$, Figure 1b) or neutrophils $\left(\mathrm{CD} 11 \mathrm{~b}^{+} \mathrm{Gr}-1^{\text {high }} \mathrm{F} 4 / 80^{-}\right.$, Figure 1c) were low (0.1\%). Frequencies of inflammatory monocytes and neutrophils increased at $\mathrm{d} 10$ and fell significantly at d21 in WT mice (Figure $\mathbf{1 b}, \mathbf{c}$ ). Conversely, the frequencies of inflammatory monocytes remained low in VDR KO mice following infection (Figure 1b). The neutrophils in VDR KO mice were significantly lower than WT mice at d 10 after infection (Figure 1c). Infection increased the frequency of $\mathrm{CD} 3{ }^{+} \mathrm{T}$ cells in the WT colonic LP at $\mathrm{d} 10$ and $\mathrm{d} 21$ after infection (Figure 1d). The increase in $\mathrm{CD} 3$ frequencies occurred after $\mathrm{d} 21$ of infection in VDR KO mice (Figure 1d). VDR KO mice had fewer $\mathrm{CD}^{+} \mathrm{T}$ cells compared with WT mice at d10 after infection (Figure 1d). The total numbers of LP lymphocytes isolated from WT and VDR KO mice were not different. The expression of Ifng, Il17a, and Il6 were low in uninfected colon from WT mice and increased significantly at d10 after infection in WT mice (Figure 1e-g). There was no increase in Ifng, Il17a, and Il6 expression with infection of VDR-KO mice (Figure 1e-g). In addition, VDR KO mice had significantly lower expression of Ifng, Il17 $a$, and Il6 than WT mice at d10 (Figure 1e-g). Lower colonization of VDR KO mice with $C$. rodentium was associated with lower numbers of immune cell infiltrates and reduced cytokine expression.

\section{Disruption of the microbiota in VDR KO mice reverses colonization resistance}

The intestinal commensal bacteria compete with $C$. rodentium for colonization. ${ }^{19}$ Previously, we had shown that VDR KO and WT mice had different commensal bacteria in the feces and that the difference in the microbiota occurred between VDR KO and WT littermates, suggesting an effect of VDR expression on the microbiota. ${ }^{20}$ Here we used quantitative real-time PCR to determine the frequencies of bacterial phyla in the feces from mice used for these experiments (Figure 2a). Confirming the published data, VDR-KO mice had decreased quantities of bacteria from the Firmicutes phylum, and increased quantities of bacteria from the Bacteroidetes and the Proteobacteria phyla in the feces compared with WT mice (Figure 2a) ${ }^{20}$ In addition, several bacterial genus members were also different in VDR KO mice compared with WT, including Eubacterium, Bacteroides, and Salmonella (Supplementary Figure S1B-D online). Similar phyla differences were also shown in the colon and small intestine (SI) from VDR KO and WT mice (Supplementary Figure S1E-J).

To determine the role of commensal bacteria in the colonization resistance of VDR $\mathrm{KO}$ mice to C. rodentium infection, $A B X$ was used to disrupt the bacteria. The one-dose $A B X$ treatment significantly reduced total amount of bacterial $16 \mathrm{~S}$ ribosomal DNA in the feces of ABX-treated WT and ABXtreated VDR KO mice compared with the values before ABX treatment (Figure 2b). In addition, ABX-treated WT and VDR KO mice had decreased bacterial diversity with $A B X$ treatment (fewer bands, Supplementary Figure S2). ABX treatment eliminated the bacterial differences in feces, colon, and SI (Supplementary Figure S1). There was no effect of ABX on the WT clearance or susceptibility to $C$. rodentium infection, and none of the ABX-treated WT mice died following infection 
Group $\times$ time: $P=0.0001$

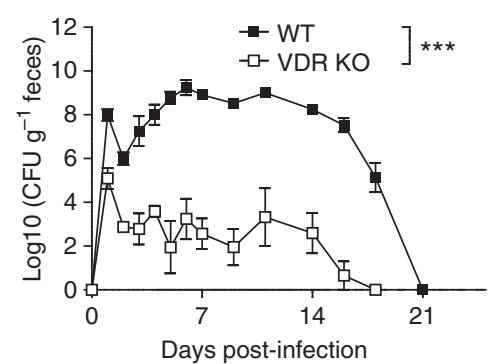

C

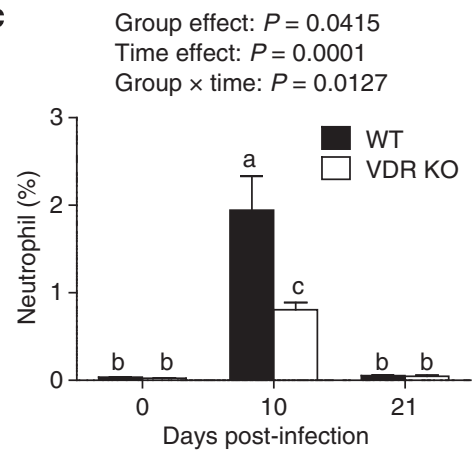

b

b

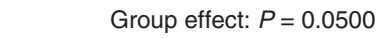

Time effect: $P=0.0007$

Group $\times$ time: $P=0.0132$

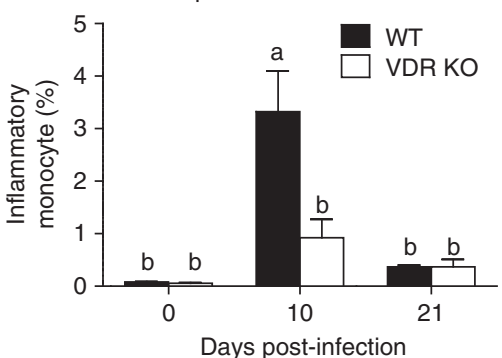

d Group effect: $P=0.0500$

Time effect: $P=0.0001$

Group $\times$ time: $P=0.0466$

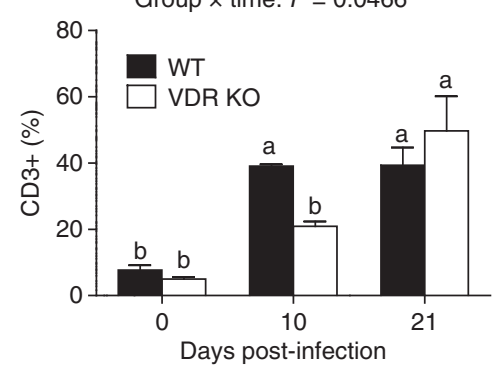

f Group effect: $P=0.021$ Time effect: $P=0.020$ Group $\times$ time: $P=0.097$

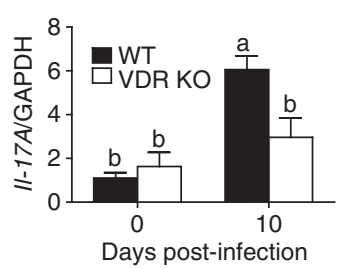

g Group effect: $P=0.124$ Time effect: $P=0.001$ Group $\times$ time: $P=0.017$

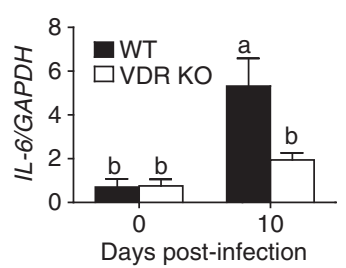

Figure 1 The kinetics of Citrobacter rodentium infection in wild-type (WT) and vitamin D receptor (VDR) knockout (KO) mice. (a) C. rodentium numbers in the feces. The frequencies of (b) inflammatory monocytes, (c) neutrophils, and (d) T cells in the colonic lamina propria (LP). mRNA expression for (e) Ifn- $\gamma$, (f) $I I-17 A$, and (g) $I I-6$ in the colon. Data are from $n=6-12$ mice per group and the values represent the mean of three independent experiments \pm s.e.m. Two-way analysis of variance (ANOVA) with Bonferroni post-hoctests $(\mathbf{a}-\mathbf{g}),{ }^{* * *} P<0.001$. Groups without a common letter differ at the indicated time point $(\mathbf{b}-\mathbf{g}), P<0.05$.

(Figure 2c, d). ABX VDR KO mice had significantly higher bacterial shedding than VDR KO mice, starting at $\mathrm{d} 2$ after infection and throughout the infection (Figure 2c). In addition, the ABX VDR KO mice took longer to clear the infection than VDR KO, ABX WT, or WT mice (Figure 2c). ABX VDR KO mice were extremely susceptible to $C$. rodentium infection and, at peak infection, 35\% died following infection (Figure 2d). $\mathrm{ABX}$ treatment reduced total bacterial numbers, changed the bacterial composition, and removed $C$. rodentium colonization resistance in VDR KO mice.

\section{Changes in the expression of antimicrobial peptides and mucin in VDR KO mice}

To determine the cause of the altered microbiota and C. rodentium colonization resistance, the expression of mRNA for several antimicrobial peptides and mucin were measured in
WT and VDR KO mice. The expression of mRNA for RegIII $\gamma$, Ang-4, mucins (Muc) 1-4, RegIII $\beta$, cathelicidin-related antimicrobial peptide, and mouse $\beta$-defensin- 3 were not different at d0 in WT and VDR KO mice (Figure 3a-c and Supplementary Figure S3). By d10, the amounts of RegIII $\gamma$, Ang-4, RegIII $\beta$, and mouse $\beta$-defensin- 3 were significantly increased in infected WT and VDR KO mice, whereas the amounts of Muc2, cathelicidin-related antimicrobial peptide, and Muc1 were only increased in infected VDR KO mice but not in infected WT mice (Figure 3 and Supplementary Figure S3). The amount of Muc3 and Muc4 were not affected by infection or genotype (Figure 3a-c and Supplementary Figure S3). At d10, VDR KO mice had higher expression levels of RegIII $\gamma$, Ang-4, and Muc2 than WT mice at d10 after infection (Figure 3a-c). ABX treatment eliminated the d10 differences in the expression for RegIII $\gamma$, Ang-4, and Muc2 between VDR KO 


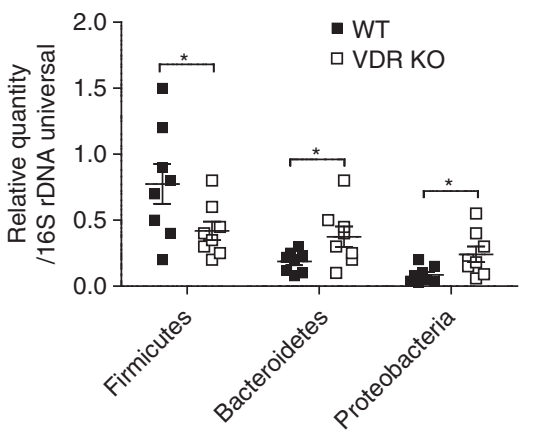

C

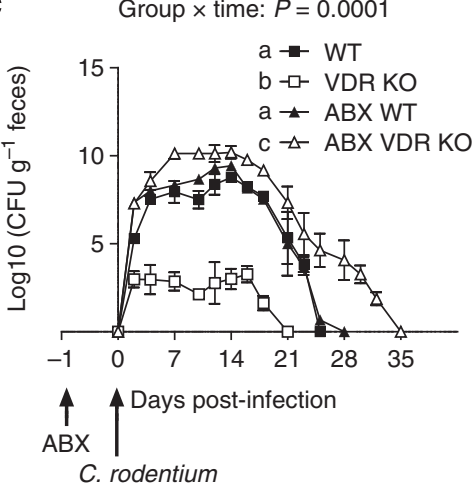

b Genotype effect: $P=0.880$

ABX effect: $P=0.001$

Genotype $\times$ ABX: $P=0.845$

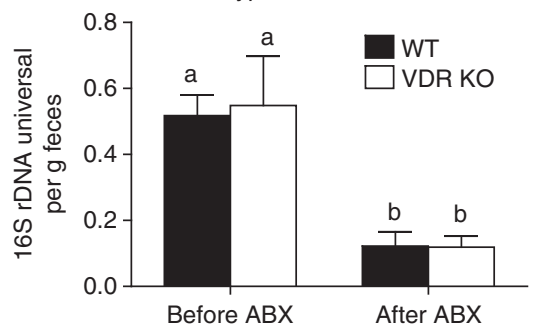

d

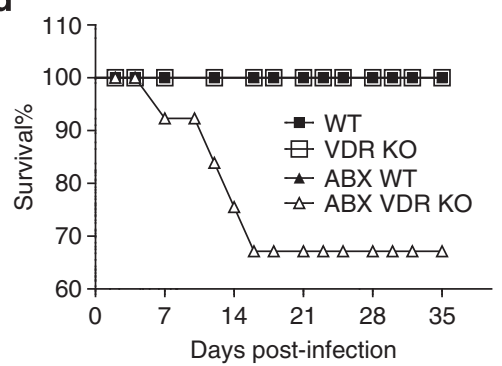

Figure 2 The effect of gut microbiota on infection with Citrobacter rodentium. (a) The relative quantity of bacteria from the Firmicutes phylum, the Bacteroidetes phylum, and the Proteobacteria phylum in the feces of wild-type (WT) and vitamin D receptor (VDR) knockout (KO) mice. (b) The relative total amount of universal 16S ribosomal DNA ( $\mathrm{rDNA}$ ) in the feces of WT and VDR KO mice before and after antibiotic (ABX) treatment. The values were normalized to the fecal weight. (c) Shedding of $C$. rodentium in the feces of WT, VDR KO, ABXWT, and ABX VDR KO mice. (d) The survival rate of the WT, VDR KO, ABX WT, and ABX VDR KO mice following infection with $C$. rodentium. Data shown are mean \pm s.e.m. using $n=4-10$ mice/group. (a) Twotailed Student's $t$-tests, (b, c) two-way analysis of variance (ANOVA) with Bonferroni post-hoc tests, and (d) log-rank test, ${ }^{*} P<0.05$. Values (b, c) without a common letter are significantly different.

a Group effect: $P=0.028$

Time effect: $P=0.001$

Group $\times$ time: $P=0.025$



Days post-infection

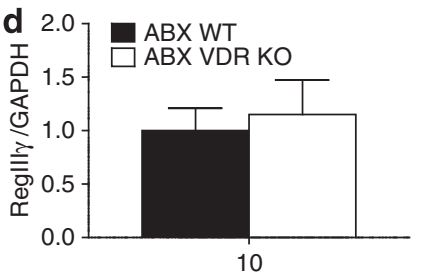

Days post-infection b

Group effect: $P=0.0402$
Time effect: $P=0.0001$

Group $\times$ time: $P=0.0425$
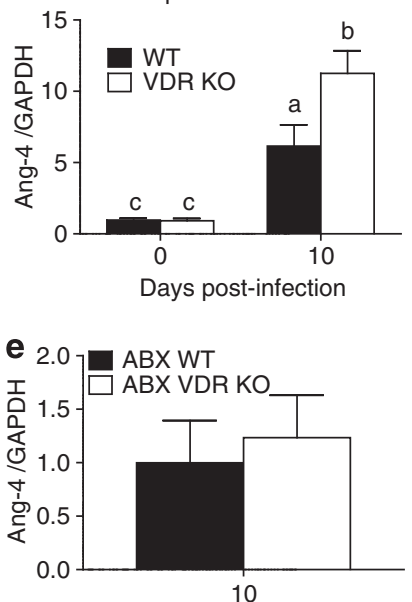

Days post-infection


Days post-infection

Figure 3 Expression of antimicrobial peptides and mucins in the colon of wild-type (WT) and vitamin D receptor (VDR) knockout (KO) mice. mRNA expression for (a, d) Reglll $\gamma$, (b, e) angiogenin-4 (Ang-4), and (c, f) mucin-2 (Muc2) in the colons of WT and VDR KO (a-c) and antibiotic (ABX)-treated WT and VDR KO (d-f) mice. Data are from $n=6-8$ mice per group and the values represent the mean of two independent experiments \pm s.e.m. $(\mathbf{a}-\mathbf{c})$ Two-way analysis of variance (ANOVA) with Bonferroni post-hoc tests and (d-f) two-tailed Student's $t$-tests. Values (a-c) without a common letter are significantly different. 
and WT mice (Figure 3a-f). VDR KO mice had increased expression of antimicrobial peptides compared with WT mice following $C$. rodentium infection and $\mathrm{ABX}$ treatment eliminated those changes.

\section{Increased IL-22 ${ }^{+}$ILCs in VDR KO mice}

IL-22 has been shown to induce epithelial cell production of antimicrobial peptides. ROR $\gamma \mathrm{t}^{+}$ILCs (predominately ILC3 cells) have been identified to be the main source of IL-22 production during the early stage of $C$. rodentium infection. ${ }^{21}$ The frequencies of ILCs $\left(\mathrm{CD} 3^{-} \mathrm{ROR} \mathrm{t}^{+}\right)$were measured in VDR KO and WT mice. There were higher frequencies of ILCs in the SI of VDR-KO mice than WT mice, but the amount of ILCs were not different in the colons of these mice (Figure 4a). In addition, VDR KO mice had more ILC3 (IL-22 producing) in the SI than WT mice (Figure 4b). Furthermore, VDR KO mice also had more $\mathrm{CD}^{-}{ }^{-} \mathrm{ROR} \gamma \mathrm{t}^{+} \mathrm{NKp}^{+} 6^{+}$(ILC1 or NK22) cells in the SI LP than WT mice (Supplementary Figure S4A). The frequencies of other ILC $\left(\mathrm{LTi}_{4}, \mathrm{CD}^{-} \mathrm{ROR} \gamma \mathrm{t}^{+} \mathrm{NKp} 46^{-}\right.$ $\mathrm{CD} 4^{+}$, and $\mathrm{LTi}_{0}, \mathrm{CD} 3^{-} \mathrm{ROR} \gamma \mathrm{t}^{+} \mathrm{NKp} 46^{-} \mathrm{CD} 4^{-}$) cells were increased in both SI and colonic LP in VDR KO mice (Supplementary Figure S4). The total cell numbers isolated from the SI or colonic LP were not different between WT and VDR-KO mice, and hence the changes in frequencies reflect the changes in absolute numbers.
A 1:1 ratio of WT and VDR KO BM was injected into lethally irradiated WT recipients. After 8 weeks, the WT recipients had $1 / 2$ of the blood and colonic lymphocytes of WT origin and the other $1 / 2$ of VDR KO origin (Figure 4c). The frequencies of ILCs in the colonic LP showed preferential reconstitution with the VDR KO ILCs compared with WT, resulting in higher ILCs of VDR KO origin in the chimeric WT mice (Figure 4d). VDR deficiency results in more IL-22-producing ILCs in the gastrointestinal tract and BM reconstitution of WT mice with VDR-KO BM demonstrates a cell-autonomous effect of the VDR on ILC frequencies in the gut.

\section{Colonization resistance is induced in WT mice by VDR KO BM}

WT BM was transplanted into WT (WT-WT) and VDR-KO (WT-VDR KO) recipients and VDR KO BM was transplanted into VDR KO (VDR KO-VDR KO) and WT (VDR KO-WT) recipients. Following confirmation of reconstitution the recipient mice were infected with $C$. rodentium. WT recipient mice receiving WT BM cells had 5 logs of $C$. rodentium at $\mathrm{d} 2$ after infection and the numbers of $C$. rodentium shed increased to 8 logs by $\mathrm{d} 7$ (Figure 4e). The VDR KO-VDR KO mice had only $4 \operatorname{logs}$ of $C$. rodentium at $\mathrm{d} 2$ after infection and the numbers remained the same at $\mathrm{d} 4$ and 7 after infection (Figure 4e). Like the VDR KO-VDR KO mice, the VDR KO-WT mice had reduced numbers of $C$. rodentium in the feces
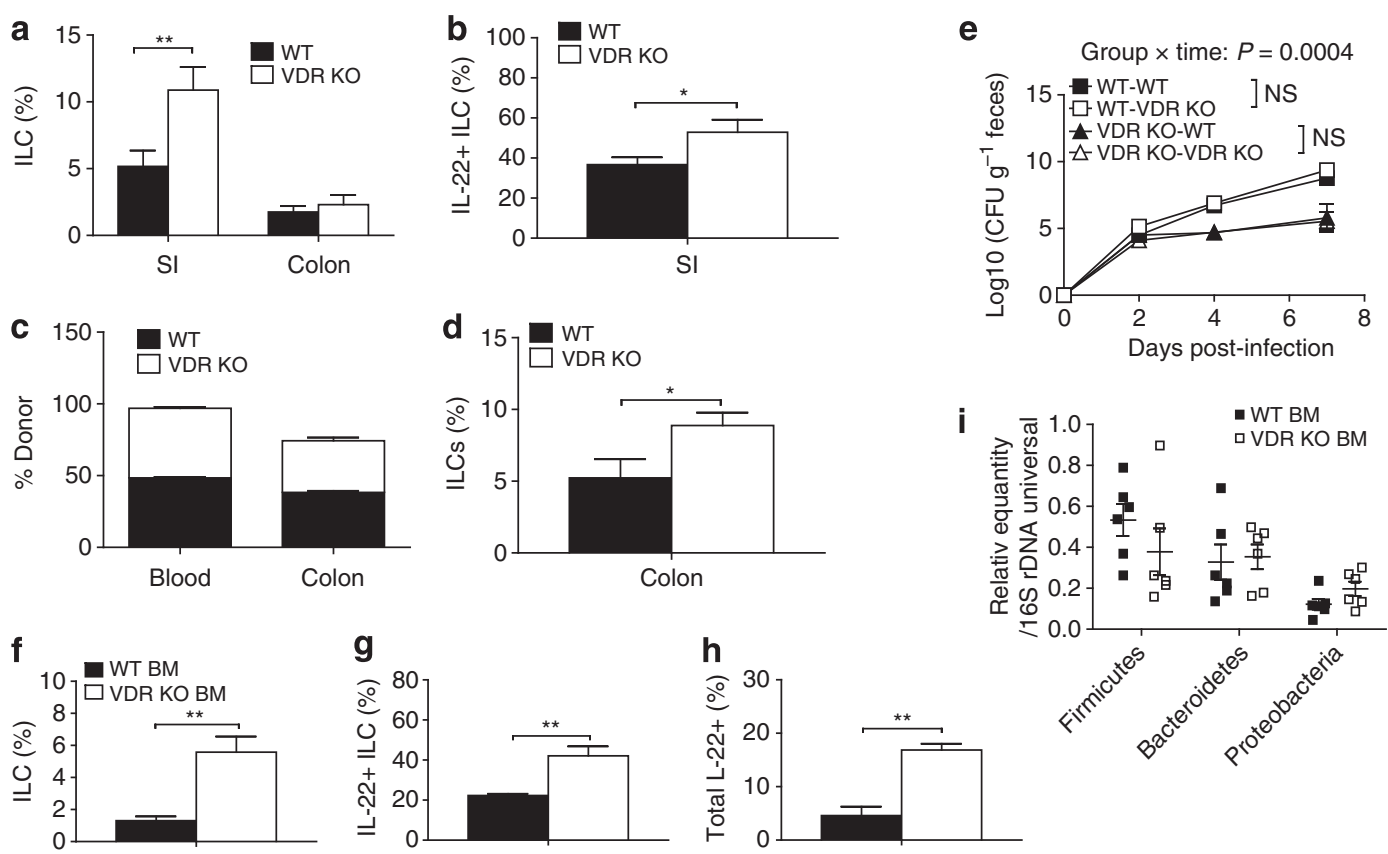

Figure 4 Cell-autonomous vitamin D receptor (VDR) control of innate lymphoid cell (ILC) number and colonization resistance. The frequencies of (a) ILCs (CD3-ROR $\gamma \mathrm{t}+$ ) and (b) interleukin-22 (IL-22)-producing ILCs in the small intestine (SI) and colon lamina propria (LP) of uninfected wild-type (WT) and VDR knockout (KO) mice. Bone marrow (BM) cells from WT, VDR KO, or a 1:1 ratio of WT and VDR KO mice were transplanted into lethally irradiated WT or VDR KO recipient mice. The frequency of VDR KO (CD45.2) and WT (CD45.1) cells in the (c) blood and colon LP and the donor-derived ILCs (d) in the colon of recipient mice. WT donor BM was transplanted into WT (WT-WT) or VDR KO recipient (WT-VDR KO) mice. VDR KO BM was transplanted into WT (VDR KO-WT) or VDR KO recipient (VDR KO-VDR KO). At 7-8 weeks after BM transplantation, recipient mice were infected with Citrobacter rodentium and the (e) colony-forming units (CFUs) in the feces were determined. The frequencies of (f) ILCs (CD3-ROR $\gamma \mathbf{t}+$ ), (g) IL-22 + ILCs, and (h) total IL-22 + cells in the SI LP of the recipients of WT BM or VDR KO BM at day 10 (d10) after $C$. rodentium infection. (i) The relative quantities of bacteria in the feces of mice 7 weeks after BM transplantation and without $C$. rodentium infection. Values are the mean \pm s.e.m. of $n=3-8$ recipient mice/group. $(\mathbf{a}-\mathbf{d}, \mathbf{f}-\mathbf{i})$ Two-tailed Student's $t$-tests and (e) two-way analysis of variance (ANOVA), ${ }^{\star} P<0.05$ and ${ }^{\star \star} P<0.01$. 
at $\mathrm{d} 4$ and $\mathrm{d} 7$ compared with the WT-WT mice (Figure 4e). Conversely, the WT-VDR KO mice had higher numbers of C. rodentium shed in the feces and the shedding resembled the WT-WT mice more than the VDR KO-VDR KO (Figure 4e). The difference in the $C$. rodentium between recipients of VDR$\mathrm{KO} \mathrm{BM}$ and WT BM was less (Figure 4e) than that between VDR-KO and WT mice (Figure 1). This may be because of incomplete reconstitution of colonic LP cells in the gut (70-75\%) of the $\mathrm{BM}$ recipients and the older age of the BM recipients (16-20 vs. 8-12 weeks) at the time of infection. However, partial colonization susceptibility and resistance to C. rodentium could be transferred by BM transplantation.

The frequencies of donor-derived ILCs from the mice in Figure 4e were measured at d10 after infection. Recipients of WT BM (both WT and VDR-KO) had fewer ILCs, fewer IL-22 + ILCs, and less total IL-22 + cells in the LP of the SI compared with recipients of VDR-KO BM (both WT and VDR-KO, Figure $\mathbf{4 f}-\mathbf{h}$ ). The frequencies of ILCs, IL-22+ ILCs, and total IL-22 + cells were significantly higher in the SI of VDR-KO BM recipients and this corresponded with colonization resistance to $C$. rodentium (Figure 4). Surprisingly, the colonization resistance was not associated with measurable changes in the bacterial phyla in the feces of the BM-reconstituted mice (Figure 4i), and this may be another reason that the colonization resistance of the VDR-KO BM recipients was less than that in the VDR-KO mice. Partial colonization resistance and susceptibility to C. rodentium was transferred via the BM. In addition, the increased numbers of IL-22-producing ILCs in the VDR-KO mice was a cellautonomous effect of VDR deficiency.

\section{Colonization resistance is transferred to GF WT mice by cecal transplants from VDR KO mice}

In order to determine whether the changes in the VDR KO microbiota could mediate colonization resistance, WT GF mice received cecal transplants from WT and VDR KO mice for $48 \mathrm{~h}$ and then were infected with $C$. rodentium. WT recipients of WT cecal contents had significantly higher fecal shedding of $C$. rodentium compared with WT recipients of VDR KO cecal contents (Figure 5a). The reduced $C$. rodentium fecal shedding in the WT recipients of VDR KO microbiota was not due to a change in ILC numbers as there was no difference in the frequencies of ILCs or IL-22 ${ }^{+}$ILCs compared with WT mice that received WT microbiota (Figure 5b,c). The WT mice with the VDR KO microbiota did have higher total IL- $22^{+}$cells in the SI than the WT recipients of WT microbiota (Figure 5d). VDR KO microbiota transferred C. rodentium colonization resistance to GF WT mice.

\section{VDR/Rag KO mice are more susceptible to $C$. rodentium than Rag KO mice}

ROR $\gamma \mathrm{t}^{+}$ILCs are also present in Rag KO mice. The uninfected Rag KO mice had 7.5\% ILCs in the SI LP that is higher than those in WT mice (5\%). ILC1, $\mathrm{LTi}_{4}$, and $\mathrm{LTi}_{0}$ cells were significantly higher in the SI (no differences in the colon) of DKO as compared with Rag KO mice (Supplementary Figure S4D-F). Despite the differences in ILC numbers between uninfected $\mathrm{DKO}$ and Rag KO mice, there were no differences in Firmicutes or Bacteroidetes phyla members in the feces from DKO and Rag KO mice (Supplementary Figure S5A). There were increased numbers of the Proteobacteria phylum in the feces from DKO mice compared with Rag KO mice (Supplementary Figure S5A). DKO and Rag KO mice were infected with $C$. rodentium to determine whether DKO mice would be colonization resistant. Bacterial shedding in Rag KO mice increased and peaked at $\mathrm{d} 7$ after infection and the Rag KO mice failed to clear the infection (Supplementary Figure S5B). Rag KO mice remained colonized with large numbers of $C$. rodentium that decreased survival beginning at d21 after infection (Supplementary Figure S5B and C). Early postinfection DKO mice had significantly higher bacterial shedding than Rag KO mice, and the DKO mice died quickly following systemic spread of the infection and weight loss (Supplementary Figure S5). DKO mice were not colonization resistant and instead were significantly more susceptible to $C$. rodentium infection than Rag $\mathrm{KO}$ mice.

\section{DISCUSSION}

VDR KO mice were found to be significantly more resistant to C. rodentium infection than WT mice. The intestinal microbiota is in competition with $C$. rodentium. A previous study demonstrated that fecal transplants from resistant mice into
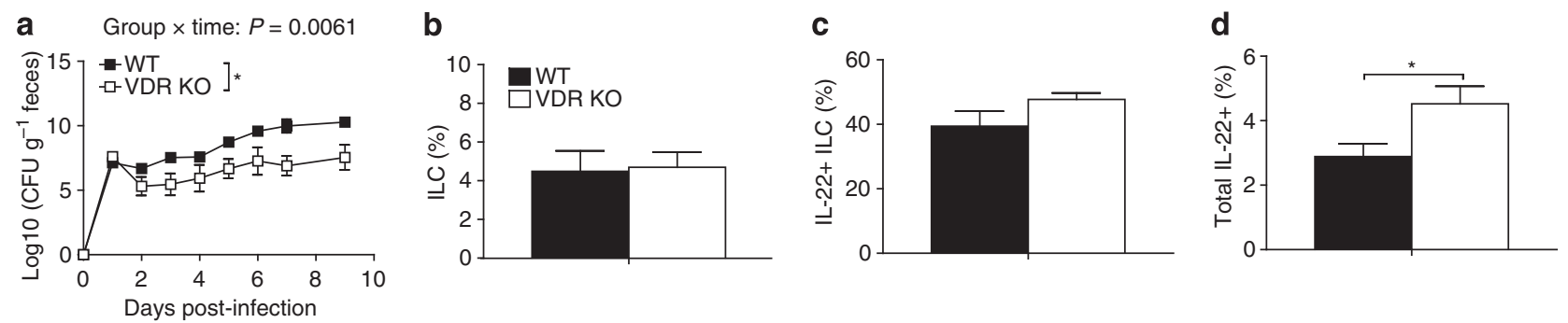

Figure 5 Cecal transplants into wild-type (WT) germ-free (GF) mice transfers colonization susceptibility to Citrobacter rodentium. Cecal contents from WT or vitamin D receptor (VDR) knockout (KO) mice were orally inoculated into WT GF mice. At $48 \mathrm{~h}$ after cecal transplantation, the WT recipient mice were infected with $C$. rodentium. (a) C. rodentium colony-forming units (CFUs) in the feces of WT GF mice receiving WT or VDR KO cecal contents. The frequencies of (b) innate lymphoid cells (ILCs) (CD3-ROR $\gamma \mathrm{t}+$ ), (c) IL-22 + ILCs, and (d) total IL-22 + cells in the small intestine (SI) lamina propria (LP) of mice in a at day 10 (d10) after $C$. rodentium infection. Values are the mean \pm s.e.m. of $n=3-8$ mice/group. (b-d)Two-tailed Student's $t$-tests and (a) one-way analysis of variance (ANOVA) with Bonferroni post-hoc tests, ${ }^{*} P<0.05$. 
susceptible mice conferred partial resistance to $C$. rodentium infection, and this protection was associated with increased IL-22 and antimicrobial peptides. ${ }^{22}$ Consistent with this study, we demonstrate that transplant of the microbiota from VDR KO mice into WT GF mice conferred protection from C. rodentium infection compared with WT microbiota. In addition, overproduction of IL-22 predominantly by ILCs in the VDR KO gut induced antimicrobial peptides that altered the microbiota in the gut. The data further demonstrate that the VDR KO microbiota induced more IL-22 than WT microbiota in GF WT recipients. However, the number of ILCs was not changed with VDR KO cecal transplant and the colonization resistance was less than that seen in the VDR KO controls. As the microbiota do not respond to changes in vitamin $\mathrm{D}$ (they do not express a vitamin $\mathrm{D}$ receptor), it seems likely that the change in IL-22 and antimicrobial peptides precedes the change in microbiota. However, our results fall short of showing a direct link between higher IL-22-producing ILCs and the shifts in the microbiota that result in colonization resistance. The VDR KO mice overproduced IL-22 and ILCs that likely caused a shift in the commensal bacteria such that the VDR KO host is resistant to infection with $C$. rodentium.

BM transplantation studies demonstrated that the higher number of ILCs in the VDR KO mouse was because of a cellautonomous effect of the VDR deficiency on ILC numbers. ILCs are critical for the control of homeostasis in the gut, but during intestinal inflammation, ILC function is dysregulated. ${ }^{23}$ In particular, ILC 3 cells that make IL-22 can be both protective and pathogenic. ${ }^{23}$ Other nutrients and the microbiota influence ILC development and function. ${ }^{23,24}$ Our results suggest that VDR regulates ILC3 cell numbers and possibly function. It might be possible that simple vitamin $\mathrm{D}$ interventions could normalize ILC function help control dysbiosis to maintain homeostasis.

Interestingly, vitamin $\mathrm{D}$ deficiency did not result in colonization resistance to C. rodentium (data not shown). ${ }^{25}$ There are several circumstances where deficiencies of the vitamin $\mathrm{D}$ ligand result in different outcomes than VDR deficiency. ${ }^{26,27}$ VDR KO BM transferred partial colonization resistance to WT mice. Conversely, WT BM transplantation into VDR KO mice resulted in $C$. rodentium colonization. The VDR is important for maintaining tight junction protein expression and barrier function in gut epithelial cells. ${ }^{28}$ Furthermore, VDR negatively regulates nuclear factor- $\kappa \mathrm{B}$ expression in gut epithelial cells. ${ }^{29}$ Our study now demonstrates an epithelial-independent effect of the VDR in controlling microbiota and IL-22 production in the gut.

VDR KO mice were extremely susceptible to $C$. rodentium infection after $\mathrm{ABX}$ treatment. $\mathrm{ABX}$ treatment disrupted the microbiota and eliminated colonization resistance. A previous study demonstrated that GF mice infected with $C$. rodentium were unable to clear the infection, suggesting the critical role of commensal bacteria in eradicating C. rodentium in the gut. ${ }^{19}$ However, none of the monoassociated GF mice died of systemic infection despite high $C$. rodentium numbers in the gut. ${ }^{19}$ Consistent with this, ABX-treated WT mice did not succumb to systemic infection. In contrast, our data showed that ABX VDR $\mathrm{KO}$ mice were unable to control $C$. rodentium and $35 \%$ died. In addition, large numbers of $C$. rodentium were detected in systemic organs of ABX VDR KO mice, including the spleen, kidney, and liver (data not shown), suggesting that VDR must be important to control infection and prevent systemic spread. $\mathrm{T}$ cells and B cells are important for containing C. rodentium in the gut. Mice deficient in T cells or B cells are unable to clear C. rodentium infection and develop lethal systemic infections. ${ }^{16}$ The increased lethality of the ABX VDR KO mice occurs within a time frame that suggests that the failure to induce a protective acquired $\mathrm{T}$ - and/or B-cell response might be preventing the VDR KO mice from containing the infection to the gut. The data suggest that acquired immunity in the VDR KO host is impaired such that once colonized the VDR KO mouse is unable or too slow in mounting a protective T/B-cell response required to clear $C$. rodentium.

The role of VDR and vitamin $\mathrm{D}$ in the control of gastrointestinal infections is complicated. Salmonella infection was more severe in VDR KO mice due, in part, to a change in nuclear factor- $\kappa \mathrm{B}$ activation in colonic epithelial cells. ${ }^{29}$ It should be noted that in order for Salmonella to infect mice, antibiotic pretreatment is required in both WT and VDR KO mice. ${ }^{29}$ Similarly, following antibiotic pretreatment here, VDR $\mathrm{KO}$ mice were more susceptible to gastrointestinal infection than WT mice. Recently, Assa et al. ${ }^{25}$ demonstrated that feeding vitamin $\mathrm{D}$-deficient diet to mice for 5 weeks was associated with increased barrier dysfunction, dysbiosis of the microbiota, and more intestinal inflammation following $C$. rodentium infection. Paradoxically, $1,25(\mathrm{OH})_{2} \mathrm{D}_{3}$ injections increased $C$. rodentium numbers in the colon and spleen of mice. ${ }^{30}$ The $1,25(\mathrm{OH})_{2} \mathrm{D}_{3}$-mediated increase in $C$. rodentium numbers was attributed to the suppression of the mucosal Th17 response. ${ }^{30}$ Our data demonstrate that VDR is a negative regulator of ILCs and infection-induced IL-22. ILCs are important for the generation of both $\mathrm{T}$ - and $\mathrm{B}$-cell responses in the gut. ${ }^{23,31}$ Interestingly, diseases like inflammatory bowel disease result in reduced expression of the VDR in the gut. ${ }^{32}$ Our data predict that with disease development the expression of the VDR will go down, creating local areas of VDR deficiency that might induce alterations in ILCs and/or dysbiosis.

Our data highlight a role for VDR in regulation of the composition of the microbial flora in the gut. Dysbiosis in the VDR KO mouse was associated with increased ILCs and IL-22producing cells in the gut that induced the production of antibacterial peptides. The increase in ILC numbers in the VDR $\mathrm{KO}$ gut is a cell-autonomous effect of the VDR. Understanding the role of vitamin D and the VDR in regulating ILC function and/or development would be critical for management of gastrointestinal homeostasis. In addition, expression of the VDR is a critical regulator of both innate and acquired immune responses as ABX VDR KO and VDR/Rag DKO mice had high numbers of $C$. rodentium in the gut that led to premature lethality of the mice following infection. VDR expression and regulation of VDR expression is critical for protection from infection and the maintenance of gastrointestinal homeostasis. 


\section{METHODS}

Mice. Age- and sex-matched WT, VDR-KO, Rag KO, and DKO mice on the C57BL/6 background were bred (breeders were VDR + / - ) and housed in the same room at the Pennsylvania State University (University Park, PA). Experimental procedures were approved by the Office of Research Protection, Institutional Animal Care and Use Committee at the Pennsylvania State University.

C. rodentium infection. The C. rodentium strain ICC169 was a kind gift of Gad Frankel (London School of Medicine and Dentistry, London, UK). C. rodentium was cultured in Luria-Bertani (LB) broth containing $20 \mu \mathrm{g} \mathrm{ml}^{-1}$ nalidixic acid (EMD Chemicals, Gibbstown, NJ). Mice were infected by oral gavage with $200 \mu \mathrm{l}$ of $C$. rodentium suspension that contained $5 \times 10^{9}$ colony-forming units. Mice were housed one per cage to prevent mouse-to-mouse transmission of $C$. rodentium. The $C$. rodentium numbers in the feces were determined by plating on LB agar plates with $50 \mu \mathrm{g} \mathrm{ml}^{-1}$ nalidixic acid. Some mice were treated with the ABX vancomycin $\left(20 \mathrm{mg} \mathrm{ml}^{-1}\right)$ the day before infection.

BM transplantation. BM cells from WT (CD45.1) and VDR KO (CD45.2) donor mice were transplanted either alone or in a 1:1 ratio into lethally irradiated WT (CD45.1/CD45.2) or VDR KO (CD45.2) recipient mice as previously described. ${ }^{33}$ At 7-8 weeks following $\mathrm{BM}$ transplantation, mice were evaluated for reconstitution in the blood (98-99\%). The reconstitution of the gut was much less $(70-75 \%$ of the total colon LP) but consistent with what has been described previously for reconstitution of the gut. ${ }^{34}$

Cecal transplant. Fresh cecal contents from one donor WT or one donor VDR KO mouse was collected and placed in phosphate-buffered saline ( $0.1 \mathrm{~g}$ cecal content per $1 \mathrm{ml}$ phosphate-buffered saline) on ice. The cecal contents from WT or VDR KO mice were homogenized and $200 \mu \mathrm{l}$ of the homogenates was orally inoculated to WT GF mice. At $48 \mathrm{~h}$ after cecal transplant, the recipient mice were orally infected with C. rodentium $\left(5 \times 10^{9}\right.$ colony-forming units per mouse).

Cell isolation and flow cytometry. Isolation of intestinal LP lymphocytes was done as described previously. ${ }^{35}$ Pieces of the SI or colon were incubated twice in Hanks' balanced salt solution containing $5 \mathrm{~mm}$ EDTA, $0.15 \mu \mathrm{g} \mathrm{ml}^{-1}$ dithiothreitol, and $5 \%$ fetal bovine serum for $20 \mathrm{~min}$ at $37^{\circ} \mathrm{C}$ under 250 r.p.m. rotation with stirring bar. The supernatant was discarded and the tissue was further incubated in RPMI1640 containing $1 \mathrm{mg} \mathrm{ml}^{-1}$ collagenase type 1 (Worthington, Lakewood, NJ) and $10 \%$ fetal bovine serum for $1.5 \mathrm{~h}$ at $37^{\circ} \mathrm{C}$ under 250 r.p.m. rotation to obtain LP cells. The LP cells were collected from the interface of 40/80\% Percoll gradients (Sigma-Aldrich, St Louis, MO). Cells were stained with fluorescein isothiocyanate (FITC) CD11b, FITC CD4, phycoerythrin (PE) Gr-1, allophycocyanin (APC) NKp46, PECy5 F4/80, PECy7 CD3, or isotype controls (eBiosciences, San Diego, CA). Sample staining histograms are shown in Supplementary Figure S6.

Cells were stimulated with phorbol 12-myristate 13-acetate $\left(0.1 \mu \mathrm{g} \mathrm{ml}^{-1}\right.$, Sigma-Aldrich) and ionomycin $\left(0.5 \mu \mathrm{g} \mathrm{ml}^{-1}\right.$, SigmaAldrich) for $5 \mathrm{~h}$, and for the final $3 \mathrm{~h}$, Brefeldin $\mathrm{A}\left(10 \mu \mathrm{g} \mathrm{ml}^{-1}\right.$, SigmaAldrich) was added to the culture medium. For the measurement of IL-22, cells were stimulated with mouse recombinant IL-23 $\left(0.04 \mu \mathrm{g} \mathrm{ml}^{-1}, \mathrm{R} \& \mathrm{D}\right.$ Systems, Minneapolis, MN) for $5 \mathrm{~h}$, and for the final $3 \mathrm{~h}$, Brefeldin A (10 $\mu \mathrm{g} \mathrm{ml}^{-1}$, Sigma-Aldrich) was added to the culture medium. After surface staining, cells were fixed with $4 \%$ paraformadehyde (Sigma-Aldrich), permeabilized with $0.1 \%$ saponin (Sigma-Aldrich), and stained with FITC interferon- $\gamma$, PE IL-17A, APC IL-22, or the FITC/PE/APC-labeled isotype controls (eBiosciences). For ROR $\gamma \mathrm{t}$, staining was done using the transcription factor staining buffer kit following the manufacturer's instructions (eBiosciences). Cells were analyzed on BD Fortessa LSRII (BD Biosciences, San Jose, CA) and the data were analyzed with FlowJo 7.6.5 software (TreeStar, Ashland, OR).

PCR. Fecal DNA was isolated using QIAamp DNA stool minikit (Qiagen, Valencia, CA). Fecal DNA was amplified with universal 16S ribosomal DNA primers or specific primers for different bacterial phyla or genus using SYBR green mix (Bio-Rad, Hercules, CA). Relative $16 \mathrm{~S}$ ribosomal DNA quantities were calculated using $\Delta \Delta \mathrm{Ct}$ method and were normalized to the amount of universal bacteria. The fecal DNA was amplified with universal 16S ribosomal DNA primers and denaturing gradient gel electrophoresis was done exactly as previously described. ${ }^{36}$

Total RNA was isolated (Qiagen). Complementary DNA was synthesized using the TaqMan reverse transcription reagents kit (Applied Biosystems, Carlsbad, CA) with SYBR green mix (Bio-Rad) by MyiQ Single-Color Real-Time PCR machine (Bio-Rad). Expression levels of these molecules were normalized by glyceraldehyde-3phosphate dehydrogenase (GAPDH) and calculated with the $\Delta \Delta \mathrm{Ct}$ method. The primer sequences are listed in Supplementary Table S1.

Statistics. Statistical analyses were performed using GraphPad software (PRISM software, La Jolla, CA). Two-tailed Student's $t$-tests were used to test differences between genotype (WT vs. KO). Two-way analysis of variance with Bonferroni post-hoc tests were used to test the effects of experimental groups, time, and their interactions. Log-rank tests were used to test the survival rates. Some of the data were transformed (square root transformation) to eliminate unequal variances, followed by a repeated-measures (mix model) two-way analysis of variance.

SUPPLEMENTARY MATERIAL is linked to the online version of the paper at http://www.nature.com/mi

\section{ACKNOWLEDGMENTS}

This study was supported by the National Institutes of Health/National Institute of Neurologic and Stroke grant NS067563, National Center for Complementary and Alternative Medicine, and the Office of Dietary Supplements AT005378.

\section{DISCLOSURE}

The authors declared no conflict of interest.

(c) 2015 Society for Mucosal Immunology

\section{REFERENCES}

1. Veldman, C.M., Cantorna, M.T. \& DeLuca, H.F. Expression of 1,25dihydroxyvitamin D-3 receptor in the immune system. Arch. Biochem. Biophys. 374, 334-338 (2000).

2. Pike, J.W. \& Meyer, M.B. The vitamin D receptor: new paradigms for the regulation of gene expression by 1,25-dihydroxyvitamin $\mathrm{D}(3)$. Endocrinol. Metabol. Clin. North Am. 39, 255-269. table of contents (2010).

3. Chiellini, G. \& DeLuca, H.F. The importance of stereochemistry on the actions of vitamin D. Curr. Top. Med. Chem. 11, 840-859 (2011).

4. Cantorna, M.T. Mechanisms underlying the effect of vitamin D on the immune system. Proc. Nutr. Soc. 69, 286-289 (2010).

5. Cantorna, M.T. et al. 1,25-Dihydroxyvitamin D3 prolongs graft survival without compromising host resistance to infection or bone mineral density. Transplantation 66, 828-831 (1998).

6. Sasidharan, P.K., Rajeev, E. \& Vijayakumari, V. Tuberculosis and vitamin D deficiency. J. Assoc. Phys. India 50, 554-558 (2002).

7. Salahuddin, N., Ali, F., Hasan, Z., Rao, N., Aqeel, M. \& Mahmood, F. Vitamin $D$ accelerates clinical recovery from tuberculosis: results of the SUCCINCT Study [Supplementary Cholecalciferol in recovery from tuberculosis]. A randomized, placebo-controlled, clinical trial of vitamin D supplementation in patients with pulmonary tuberculosis'. BMC Infect. Dis. 13, 22 (2013).

8. Martineau, A.R., Honecker, F.U., Wilkinson, R.J. \& Griffiths, C.J. Vitamin D in the treatment of pulmonary tuberculosis. J. Steroid Biochem. 103, 793-798 (2007).

9. Wejse, C. et al. Vitamin D as supplementary treatment for tuberculosis: a double-blind, randomized, placebo-controlled trial. Am. J. Resp. Crit. Care. Med. 179, 843-850 (2009).

10. Liu, P.T. et al. Toll-like receptor triggering of a vitamin D-mediated human antimicrobial response. Science 311, 1770-1773 (2006) 
11. Hewison, M. et al. Extra-renal 25-hydroxyvitamin D3-1alpha-hydroxylase in human health and disease. J. Steroid Biochem. Mol. Biol. 103, 316-321 (2007).

12. Froicu, M., Weaver, V., Wynn, T.A., McDowell, M.A., Welsh, J.E. \& Cantorna, M.T. A crucial role for the vitamin D receptor in experimental inflammatory bowel diseases. Mol. Endocrinol. 17, 2386-2392 (2003).

13. Ehrchen, J. et al. Vitamin D receptor signaling contributes to susceptibility to infection with Leishmania major. FASEB J. 21, 3208-3218 (2007).

14. Bruce, D., Whitcomb, J.P., August, A., McDowell, M.A. \& Cantorna, M.T. Elevated non-specific immunity and normal Listeria clearance in young and old vitamin D receptor knockout mice. Int. Immunol. 21, 113-122 (2009).

15. Luperchio, S.A. \& Schauer, D.B. Molecular pathogenesis of Citrobacter rodentium and transmissible murine colonic hyperplasia. Microb. Infect. 3 , 333-340 (2001).

16. Mundy, R., MacDonald, T.T., Dougan, G., Frankel, G. \& Wiles, S. Citrobacter rodentium of mice and man. Cell Microbiol. 7, 1697-1706 (2005).

17. Rubino, S.J., Geddes, K. \& Girardin, S.E. Innate IL-17 and IL-22 responses to enteric bacterial pathogens. Trends Immunol. 33, 112-118 (2012).

18. Sonnenberg, G.F., Monticelli, L.A., Elloso, M.M., Fouser, L.A. \& Artis, D. $\mathrm{CD} 4(+)$ lymphoid tissue-inducer cells promote innate immunity in the gut. Immunity 34, 122-134 (2011).

19. Kamada, N. et al. Regulated virulence controls the ability of a pathogen to compete with the gut microbiota. Science 336, 1325-1329 (2012).

20. Ooi, J.H., Li, Y., Rogers, C.J. \& Cantorna, M.T. Vitamin D regulates the gut microbiome and protects mice from dextran sodium sulfate-induced colitis. J. Nutr. 143, 1679-1686 (2013).

21. Zheng, Y. et al. Interleukin-22 mediates early host defense against attaching and effacing bacterial pathogens. Nat. Med. 14, 282-289 (2008).

22. Willing, B.P., Vacharaksa, A., Croxen, M., Thanachayanont, T. \& Finlay, B.B. Altering host resistance to infections through microbial transplantation. PLoS One 6, e26988 (2011).

23. Sonnenberg, G.F. Regulation of intestinal health and disease by innate lymphoid cells. Int. Immunol. 26, 501-507 (2014).

24. van de Pavert, S.A. et al. Maternal retinoids control type 3 innate lymphoid cells and set the offspring immunity. Nature 508, 123-127 (2014).
25. Assa, A., Vong, L., Pinnell, L.J., Avitzur, N., Johnson-Henry, K.C. \& Sherman, P.M. Vitamin D deficiency promotes epithelial barrier dysfunction and intestinal inflammation. J. Infect. Dis. 210, 1296-1305 (2014).

26. Mayne, C.G., Spanier, J.A., Relland, L.M., Williams, C.B. \& Hayes, C.E. 1,25-Dihydroxyvitamin D3 acts directly on the $T$ lymphocyte vitamin $D$ receptor to inhibit experimental autoimmune encephalomyelitis. Eur. J. Immunol. 41, 822-832 (2011).

27. Driver, J.P., Lamont, D.J., Gysemans, C., Mathieu, C. \& Serreze, D.V. Calcium insufficiency accelerates type 1 diabetes in vitamin $D$ receptordeficient nonobese diabetic (NOD) mice. Endocrinology 152, 4620-4629 (2011).

28. Kong, J. et al. Novel role of the vitamin D receptor in maintaining the integrity of the intestinal mucosal barrier. Am. J. Physiol. Gastrointest. Liver Physiol. 294, G208-G216 (2008).

29. Wu, S. et al. Vitamin D receptor negatively regulates bacterial-stimulated NF-kappaB activity in intestine. Am. J. Pathol. 177, 686-697 (2010).

30. Ryz, N.R. et al. Active vitamin D (1,25-dihydroxyvitamin D3) increases host susceptibility to Citrobacter rodentium by suppressing mucosal Th17 responses. Am. J. Physiol. Gastrointest. Liver Physiol. 303, G1299-G1311 (2012).

31. Tsuji, M. et al. Requirement for lymphoid tissue-inducer cells in isolated follicle formation and T cell-independent immunoglobulin A generation in the gut. Immunity 29, 261-271 (2008).

32. Liu, W. et al. Intestinal epithelial vitamin D receptor signaling inhibits experimental colitis. J. Clin. Invest. 123, 3983-3996 (2013).

33. Yu, S. \& Cantorna, M.T. The vitamin D receptor is required for iNKT cell development. Proc. Natl. Acad. Sci. USA 105, 5207-5212 (2008).

34. Bruce, D. \& Cantorna, M.T. Intrinsic requirement for the vitamin D receptor in the development of CD8alphaalpha-expressing T cells. J. Immunol. 186, 2819-2825 (2011).

35. Weigmann, B., Tubbe, I., Seidel, D., Nicolaev, A., Becker, C. \& Neurath, M.F. Isolation and subsequent analysis of murine lamina propria mononuclear cells from colonic tissue. Nat. Protoc. 2, 2307-2311 (2007).

36. Muyzer, G., Dewaal, E.C. \& Uitterlinden, A.G. Profiling of complex microbial-populations by denaturing gradient gel-electrophoresis analysis of polymerase chain reaction-amplified genes-coding for 16S rRNA. Appl. Environ. Microb. 59, 695-700 (1993). 